\title{
Cooperative-Intelligent Transport Systems for Vulnerable Road Users safety
}

\author{
Jordi Casademont \\ Universitat Politècnica de Catalunya \\ i2CAT Foundation \\ Barcelona, Spain \\ Mònica Navarro \\ CTTC \\ Castelldefels, Spain
}

\author{
Anna Calveras \\ Universitat Politècnica de Catalunya \\ Barcelona, Spain \\ Javier Arribas \\ CTTC \\ Castelldefels, Spain
}

\author{
David Quiñones \\ i2CAT \\ Barcelona, Spain
}

Miguel Catalán-Cid
i2CAT
Barcelona, Spain

\begin{abstract}
Cooperative-Intelligent Transport Systems (CITS) are one of the flagships of new coming automotive and communication industries. Mobility needs to be safer and more efficient and C-ITS are the key for this new era. On the one hand, organizations like ETSI, IEEE, SAE, 3GPP or ISO are developing the standards for the new technologies and protocols and, on the other, manufacturers and operators are deploying and testing their first pilots. In this paper, we present a pilot developed by a group of stakeholders, in which vehicles will alert drivers of potential collisions with vulnerable road users riding bicycles. It is a multidisciplinary project where there are different architecture components: C-ITS stations integrated in vehicles, vehicles provided with digital cockpits that show warning messages to the driver, low-cost C-ITS stations attached to bicycles which are equipped with a high precision location system based on the fusion of different information sources as GPS, inertial sensors and Ultra Wide Band ranging and finally communication between C-ITS stations is provided by a network that supports low delay C-V2X communications with a Multi-access Edge Computing which takes routing decisions.
\end{abstract}

Keywords-C-ITS, VRU, Safety, LTE-V2V, 5G, Multi-access Edge Computing, MEC. location techniques, UWB,

\section{INTRODUCTION}

Cooperative - Intelligent Transport Systems (C-ITS) are the next step to increase safety and efficiency in road transport. They can make driving safer, decrease traffic jams and improve oil consumption, as well as enable any kind of Internet service to be consumed inside the car.

Nowadays, cars come with sensors which enable them to be aware of their surroundings and other cars moving around, and with cameras which can read traffic signals and detect the paintings on the road; but what would it happen if the dangerous event is not in the range of the sensors or out of view of the cameras? What would it happen if the third or fourth car ahead suddenly brakes? How much reaction time the driver is going to lose before noticing the event? Basically, answers are that car's sensors and cameras cannot detect events which are at Non-Line of Sight (NLOS), and the driver will lose a vital time before breaking, which could lead to a collision..

To improve these technologies, the next stage is to provide communication between vehicles. V2X Communications (Vehicle to Everything) enable cars to share their position, speed, heading and even data sensed by their own sensors or cooperative instructions. Vehicles can exchange information with other vehicles (V2V), with the roadside infrastructure
(V2I), with a backend server (e.g., from a vehicle manufacturer or traffic centres) or with Vulnerable Road Users as pedestrians or bike riders.

Vehicle communication is a key enabler for the provision of value-added services related to driving safety (collision risk warning, emergency electronic brake lights notification ...), traffic efficiency (traffic light optimal speed advisory, invehicle signage, cooperative merging ...) or additional services (point of interest notification, media downloading ...) [1].

Nevertheless, many of these new services require a localization information of high accuracy which, at present time, GNSSs (Global Navigation Satellite Systems) are not able to provide at civil sector by themselves. Therefore, the evolution of communications between vehicles requires a further progress in location systems. One option is to combine different mechanisms and fusion their outcome in a higher precision location. For instance, in our proposal we have combined a positioning system based on GPS (Global Position System), inertial sensors and distance measures from an UWB (Ultra Wide Band) ranging system. Using this method, we get the so-called lane-accuracy precision which enables to successfully deploy C-ITS.

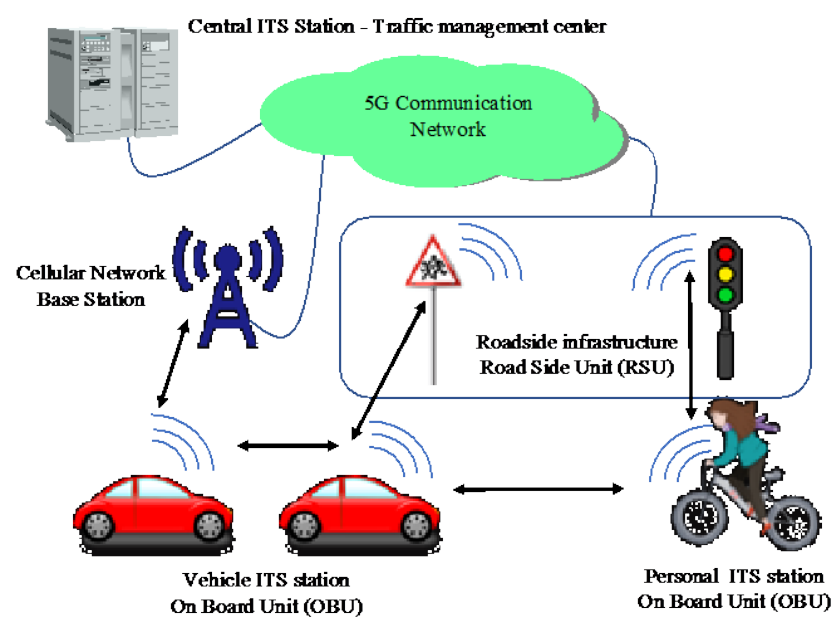

Fig. 1. C-ITS infrastructure elements.

The deployment of C-ITS needs a multi-stakeholder cooperation (Fig. 1). Firstly, V2X equipment manufacturers of devices deployed nearby the road (Road Side Unit - RSU) and devices in cars (On Board Unit - OBU). Secondly, cars manufacturers that integrate OBUs in their vehicles. Thirdly, operators which deploy RSUs in the territory and connect them to the main communication network. Nevertheless, 
sometimes there will be no RSU coverage in certain areas and OBUs can connect directly to base stations of cellular operators which provide communications C-V2X (Cellular Vehicle to Everything). Then, as an additional service, it is possible to add a certain level of intelligence in the infrastructure, which can be at traffic management centres or closer to the vehicles, near the RSUs, as Multi-Access Edge Computing (MEC) that can provide much faster responses in critical situations. Finally, it is necessary a very low latency communication network as the so-called 5G technology emerging nowadays.

In this paper we present a pilot, showcased in Mobile World Congress 2019, of a system that prevents collisions between cars and bicycles. For one hand, CTTC (Centre Tecnològic Telecomunicacions Catalunya) and i2CAT have developed a high precision location system fusioning data from GPS, inertial sensors and UWB ranging system. This equipment has been connected to a Raspberry Pi 3 running Vanetza framework [2], which implements ETSI (European Telecommunications Standards Institute) C-ITS protocol stack, and with a $\mathrm{C}-\mathrm{V} 2 \mathrm{X}$ communication dongle form the OBU attached to the bicycle. This OBU continuously transmits its position using CAMs (Cooperative Awareness Message) which are received by a base station of Movistar who has deployed a MEC nearby it. The MEC forwards CAMs to their destination using again C-V2X communications. Vehicles from SEAT are provided with OBUs manufactured by Ficosa which receive these CAMs and analyse potential collision situations. In case of danger, a warning message is displayed in driver's cockpit.

The paper is organized as follows. Section II presents the general architecture for C-ITS, its protocol architecture and radio technologies. Section III introduces the problematic of acquiring a precise location with GNSS and describes the adopted solution. Section IV presents the pilot developed by the consortium. Finally, Section V concludes the paper.

\section{COOPERATIVE INTELLIGENT TRANSPORT SystemS}

Nowadays, there are several communication protocols architectures, which, more or less, agree on the same structure that is designed on four different protocol layers elements (Fig. 2).

\section{A. Application layer:}

It provides end user applications. As for the presented pilot, the bicycle's OBU collects its accurate location and sends it to the lower layer's Cooperative Awareness service. The car's OBU receives the bicycle position and computes if there is a dangerous situation to warn the driver.

\section{B. Facilities layer:}

It provides support functions for applications and the definition of standard messages that can be used by different applications. In this way, the number of used messages is maintained low although the number of applications could be large. The two basic messages for safety related services are:

- CAM (Cooperative Awareness Message) is a message exchanged between ITS stations to create and maintain awareness of each other. A CAM contains status and attribute information of the originating ITS station as station type (pedestrian, cyclist, motorcycle, passenger car, bus, light truck, tram, Road Side Unit ...), position, heading, speed, acceleration, lane position, drive direction, vehicle length and width ... [3].

- DENM (Decentralised Environmental Notification Message) is mainly used by ITS applications in order to alert road users of a detected event as accident, roadworks, adverse weather condition (visibility, precipitation), obstacle/animal/human on the road, wrong way driving, slow vehicle, vehicle breakdown, post-crash, emergency vehicle approaching, dangerous curve, collision risk, signal violation ... [4]

\section{Networking and transport layer:}

Networking and transport layer provides data routing functions that can be single-hop or multi-hop, identification of source and destination applications (using a port identification system), data congestion management and error control. In the present pilot we have used ETSI protocols: GeoNetworking (GN) with single-hop broadcast addressing mode and Basic Transport Protocol (BTP)

\section{Access technologies layer:}

It provides radio Medium Access Control (MAC) and physical levels. It allows different types of radio technologies.

Up to present day, IEEE 802.11 p has been the de facto wireless technology standard for V2X communications since its release in 2010. It is the former access technology of the Wireless Access in Vehicular Environment (WAVE) protocol architecture in the US, and it has also been adopted in the ETSI ITS-G5 for Europe. It is a relatively mature technology and has already been validated by over a decade of field trials. Nevertheless, theoretical and simulation studies show high level of errors under heavy traffic conditions [5], [6].

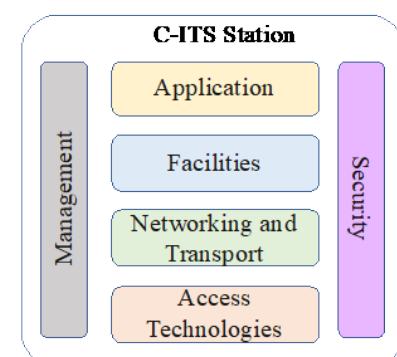

Fig. 2. C-ITS communication protocols architecture.

Long-Term Evolution (LTE) based V2X from the Third Generation Partnership Project (3GPP) is a relatively new alternative to IEEE $802.11 \mathrm{p}$. The first version of LTE-V2X was published in June 2017 under Release 14, which came with numerous enhancements to the existing Device-toDevice (D2D) communications in order to accommodate vehicular communications. The proposed enhancements include a new arrangement of the resource grid of the physical layer, and two types of V2V channel access mechanisms: i) a mechanism coordinated by the evolved NodeB (eNB), named Mode 3, and ii) a distributed mechanism, where User Equipments (UEs) access the channel on their own, named Mode 4. Moreover, LTE-V2X employs different radio interfaces: i) interface between the vehicle and eNB, named LTE-Uu, and ii) interface between vehicles, named PC5. PC5 is intended for transmission of messages belonging to C-ITS applications. Uu is used for vehicles to request transmission resources on PC5 channel to eNB, and for eNB to grant these resources to vehicles, so the cellular network selects and manages the radio resources used by vehicles for their direct 
$\mathrm{V} 2 \mathrm{~V}$ communications, enabling to reduce collisions at maximum. Mode 4 includes a distributed scheduling scheme for vehicles to select their radio resources.

In IEEE $802.11 \mathrm{p}$ messages are transmitted according to a Carrier-Sense Multiple Access with Collision Avoidance (CSMA/CA) algorithm, where only a single frame can be transmitted at the time. On the contrary LTE-V2X uses an Orthogonal Frequency-Division Multiple Access (OFDMA) structure that enables to simultaneously transmit several messages arranged in a resource grid over PC5 channel.

According to recent studies like [5] and [6], LTE-V2X higher flexibility contributes to a better reliability and range with respect to IEEE 802.11 p.

\section{LOCATION FOR C-ITS}

Accurate and robust location is essential to C-ITS services and applications. There exist a wide range of technologies none of which provides the optimal solution for the wide range of scenarios emerging in C-ITS and vehicular communications (e.g. see the challenges identified in [7]). In general, the approach is to provide a multi-sensor hybrid solution, which combines them to use the best of each technology to obtain a single blended solution for position, velocity and, in some cases, altitude estimation.

In this work, we focus on GNSS-based positioning techniques, since it is a low-cost technology with a wide market penetration and it provides an absolute reference location. Both are relevant features in the context of C-ITS and optimization of traffic management, especially in high density environments such as cities. Although GNSS can reach very accurate positioning, in particular with good satellite visibility and enhanced using reference GNSS ground stations corrections in combination with dual-band receivers (which chipsets have recently been reached the market [8]), the widely used GNSS receivers still suffer from vulnerable conditions that can lead to denial of service or result in unacceptable errors for C-ITS services. These performance degradations are related to poor satellite visibility in urbancanyons or even complete loss of signal such in tunnels, multipath or ionosphere scintillation.

It has been shown that multi-sensor fusion techniques can combat these vulnerabilities $[9,10]$. In particular, in previous work we developed a hybrid GNSS/INS prototype, which also ca incorporated additional ranging or positioning measurements from complementary technologies [11]. The fusion algorithm is technology agnostic; however, we consider a solution based on high resolution radio ranging, such as the one provided by UWB technology. The motivation is to complement, with additional low cost infrastructure, urban scenarios with challenging conditions, focusing on increasing safety for vulnerable road users, and targeting lanelevel accuracy. We next describe the prototype components and the implemented fusion algorithm for real-time multisensor measurements fusion.

\section{A. HYBRID GNSS/INS/UWB PROTOYPE}

Fig. 3 shows the complete prototype and the specific hardware placement used during the demonstration. It consists of the hybrid positioning hardware prototype (implemented in the top raspberry), the developed communication interface (implemented in the bottom raspberry), the commercial cellular communication dongle, the UWB transceiver acting as tag, the GPS antenna and the power bank battery.

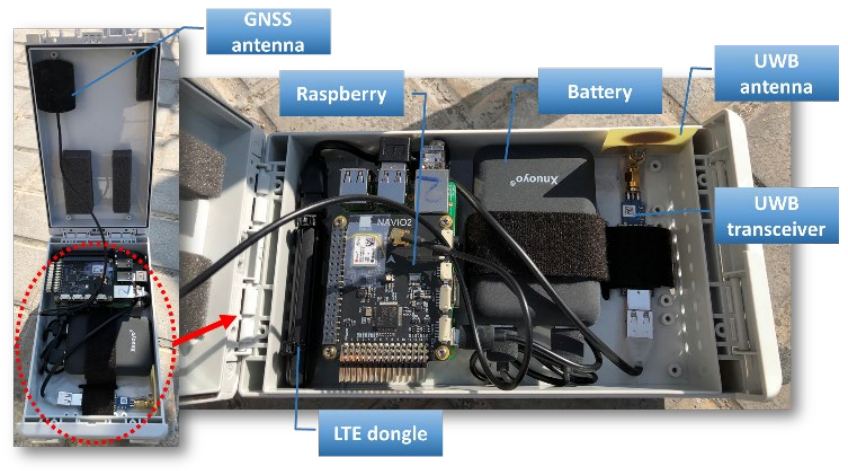

Fig. 3. Hybrid GNSS/INS/UWB positioning prototype.

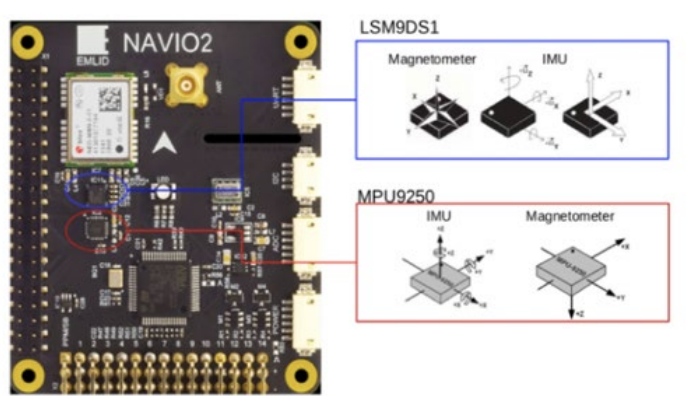

Fig. 4. Details of NAVIO2 IMU board (Emlid Ltd).

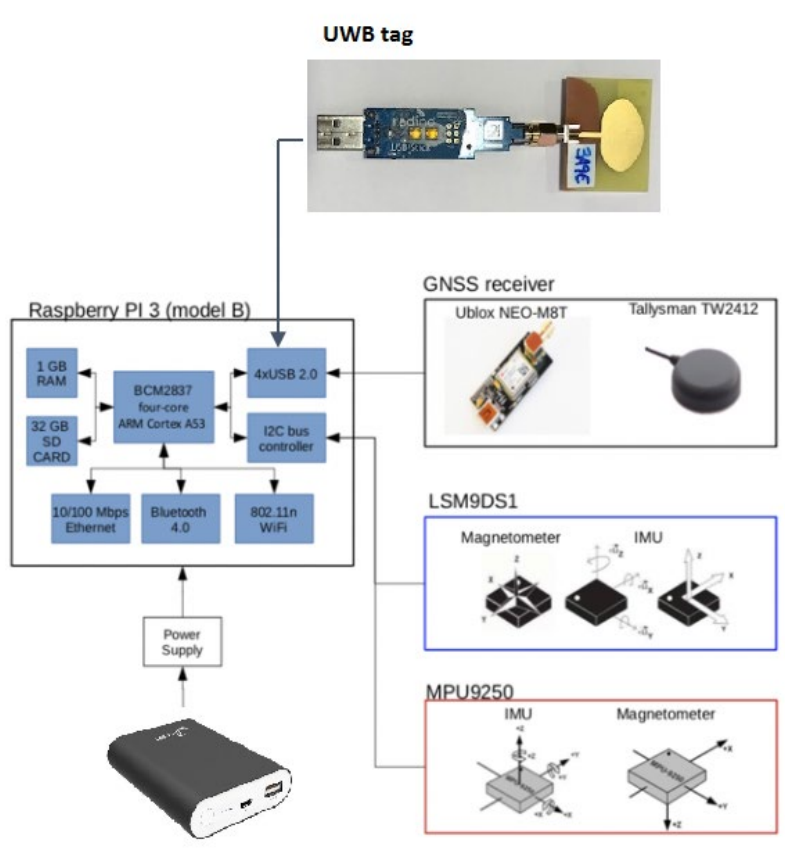

Fig. 5. Schematic fusion prototype inputs and interfaces.

The multi-sensor data fusion prototype is based on:

- CPU module implemented using a Raspberry PI 3 model B board, which provides the necessary computing power for the multi-sensor data fusion algorithm.

- NAVIO2 IMU (Inertial Measurement Unit) board (Emlid Ltd), which contains 2 low-cost IMUs with a 3-axis accelerometer, 3-axis gyroscope and 3-axis magnetometer (Fig. 4). 
- GNSS receiver (model UBLOX NEO-M8M), which provides measurements at several rates (it can be selected to operate as low as $1 \mathrm{~Hz}$ ). The GNSS is used to provide precise time-stamping to the other sensor measurements (IMU and UWB).

- UWB tag providing ranging measurements if available.

Fig. 5 provides more details on the CPU modules (Raspberry $\mathrm{Pi}$ ) and the port interfaces with the sensors used for the data fusion. The hybrid sensor fusion algorithm is implemented in the Rapsberry Pi, displayed in Fig. 3. Due to hardware integration issues, the communication interface software which takes the WGS84 positions delivered by the positioning prototype and converts them to the suitable CAM message format to be delivered to the cellular network was required to be implemented on a second Raspberry.

\section{B. UWB ranging system}

The used device for UWB ranging is the radino32, that integrates a DW1000 Integrated Circuit chip from DecaWave. It brings decimetre level positioning as an opportunity in location systems and can complement other system approaches.

The key features of this technology can be summarized as follows: it allows high localization precision, it has no interference with other radio communication systems, it is resistant to multipath signal propagation and noise, and it uses low power transceivers.

Unlike traditional radio technologies (like Bluetooth or Wi-Fi), UWB operates with the signal's so-called Time of Flight (ToF) rather than Received Signal Strength Indication (RSSI), which makes the technology much more precise and enables it to obtain very exact ranging measurements. Distance is obtained as:

$$
\text { Distance }=\mathrm{ToF}^{*} \text { speed of light }
$$

The ToF is calculated using the Decawave Two Way Ranging protocol between two modules based on UWB IEEE 802.15.4a standard. The so-called Tag is the device we want to locate, and the Anchor is the device that acts as reference. There are three messages exchanged between Tag and Anchor in order to get a precise measure of the ToF: Poll, Response and Final. ToF and distance are calculated on the Anchor, based on Tag and Anchor timestamps. Finally, Report message might be employed in order to transfer distance measurement from the Anchor back to Tag as in Fig. 6.

$\mathrm{ToF}=\left(\left(\mathrm{T}_{\mathrm{RR}}-\mathrm{T}_{\mathrm{SP}}\right)-\left(\mathrm{T}_{\mathrm{SR}}-\mathrm{T}_{\mathrm{RP}}\right)+\left(\mathrm{T}_{\mathrm{RF}}-\mathrm{T}_{\mathrm{SR}}\right)-\left(\mathrm{T}_{\mathrm{SF}}-\mathrm{T}_{\mathrm{RR}}\right)\right) / 4$

DW1000 Radinos have many configuration parameters, in our case, we have configured them in the following way:

- Power: $33,5 \mathrm{dBm}$.

- Channel: 4.

- TX rate: $110 \mathrm{Kbps}$.

- TX pulse frequency: $64 \mathrm{MHz}$.

- Preamble length: 1024 bits.

- Preamble code: 17.

- Short Address is directly read from EEPROM memory.

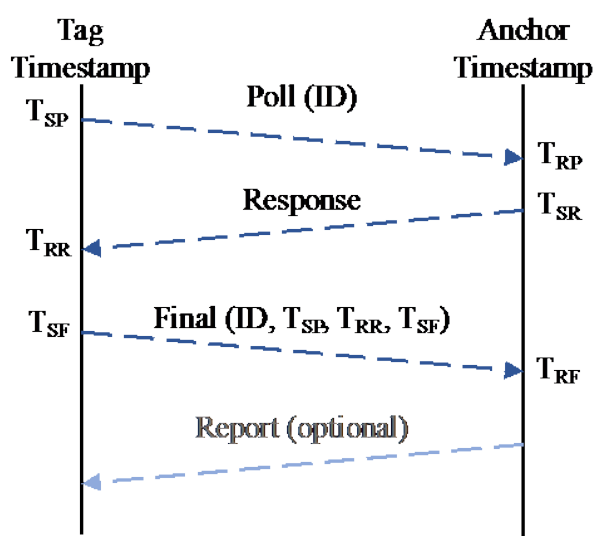

Fig. 6. UWB ranging protocol.

\section{Fusion}

The sensor fusion algorithm is based on the strapdown Inertial Navigation System (INS) architecture with a loose GNSS/INS integration. A detailed description of the loose and tight integration of the GNSS/INS hybrid position scheme can be found in $[11,12]$.

The motivation for choosing the loose/tight hybrid integration in this work is two-fold: on the one hand one can fuse directly the observable GNSS (position, velocity and time, known as loosely integration) provided by all commercial GNSS receivers, without the need to access to GNSS raw measurements, which are in general available in a less number of chipsets or devices and also typically more expensive. On the other hand, the tightly integration of distance measurements requires less heavy external infrastructure, that is a less number of UWB anchors. Considering only the ranging measurements, and not the positioning provided by the UWB devices, allows the system to take advantage of just one UWB ranging, without the need to at least see three anchors to solve the two-dimensional position problem.

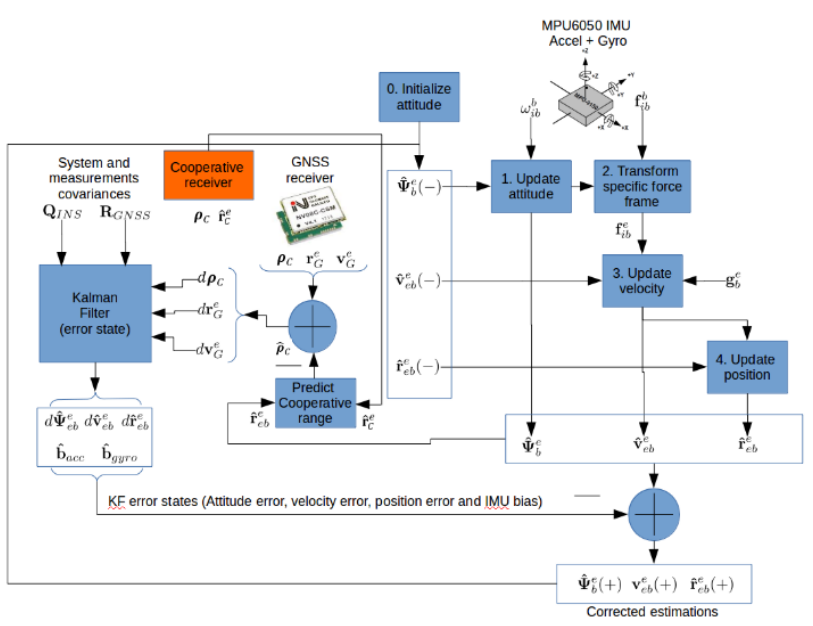

Fig. 7. Hybrid GNSS/INS/UWB fusion algorithm block-diagram.

This approach also relaxes constraints on radio access protocol, which is particularly beneficial in scenarios of moderate to high velocity. The other observations taken by the data fusion algorithm are the INS acceleration forces and angular velocity provided by the IMU. Notice that both observations must be expressed in the same reference frame, so the INS solution must be converted from body to ECEF (Earth-centred Earth-fixed). The other observation is the 
relative distance between the UWB tag and the anchors. It is relevant to remark that GNSS, INS and UWB measurement rates may be different. The fusion algorithm takes it into account and, outputs a solution whenever they are available. However, the output rate is governed by the IMU measurement rate.

The algorithm also integrates the GNSS velocity observable to estimate the heading, since the INS heading estimates are not very reliable at low vehicle dynamics as it can be the case for the bicycle or pedestrian users.

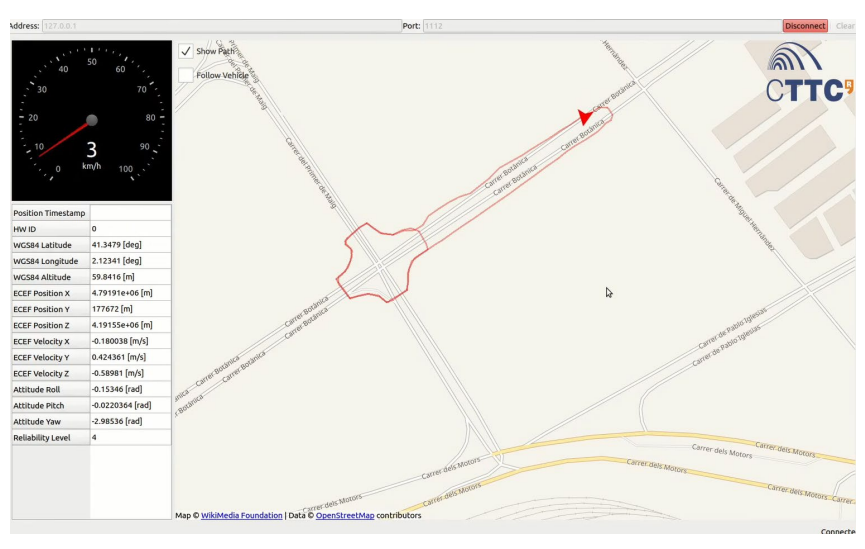

Fig. 8. Route followed by the bicycle resulting from the hybrid positioning algorithm

\begin{tabular}{|c|c|c|c|}
\hline $\begin{array}{c}\text { UWB } \\
\text { anchor }\end{array}$ & X Coord & Y Coord & Z Coord \\
\hline 1 & 426468.775 & 4577670.042 & 8.432 \\
\hline 2 & 426478.791 & 4577649.907 & 7.963 \\
\hline 3 & 426497.813 & 4577640.361 & 8.203 \\
\hline 4 & 426495.282 & 4577661.620 & 7.969 \\
\hline 5 & 426509.656 & 4577653.800 & 8.064 \\
\hline 6 & 426536.462 & 4577675.084 & 8.060 \\
\hline 7 & 426560.818 & 4577675.956 & 8.409 \\
\hline 8 & 426584.669 & 4577706.956 & 7.460 \\
\hline
\end{tabular}

Fig. 9. GPS location of the UWB anchors.

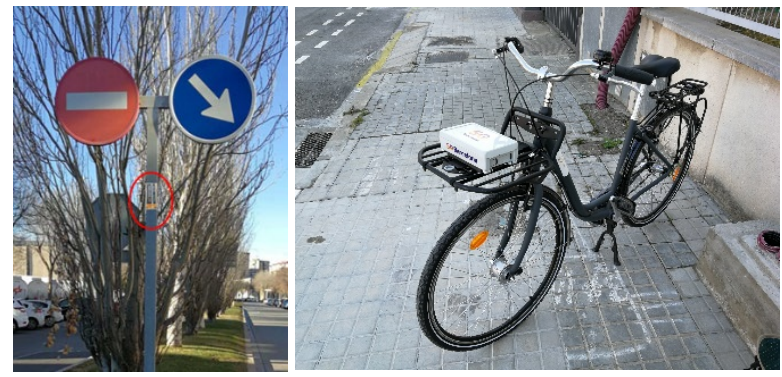

Fig. 10. UWB anchor and bicycle positioning prototype.

\section{BICYCLE DETECTION TESTBED}

A pilot showcase was deployed during the Barcelona's Mobile World Congress 2019 to validate the capacity of the deployed prototype to prevent collisions between bicycles and cars.

\section{A. Testbed setup and scenario}

Fig. 8 shows the location of the pilot and the route followed by the bicycle during the experiments. A total of eight UWB anchors powered by batteries were installed at a height of $2.5 \mathrm{~m}-3 \mathrm{~m}$ in the area to provide coverage to the location system. The GPS position of the UWB anchors was exactly measured by a surveyor to minimize the errors of the location algorithm (see Fig. 9 and Fig. 10 left). Furthermore, the positioning prototype was installed in the front of a bicycle, as shown in Fig. 10 right.

As commented previously, the main board of the prototype implements the C-ITS protocol stack (Vanetza framework) and is configured to report location updates every $100 \mathrm{~ms}$ using CAM messages to a MEC expressly deployed for the pilot. Finally, the demonstration car enabled with an OBU, receives this information and determines if there exists a potential collision danger. For this, "danger zones" for car and bicycle were defined.

\section{B. Results}

During the days of the testbed, about sixty iterations of the bicycle detection experiment were performed. In all the cases, when the bicycle entered the danger zone of the car a notification was displayed in the driver's cockpit as illustrated in Fig. 11, demonstrating the feasibility of the collision warning service.

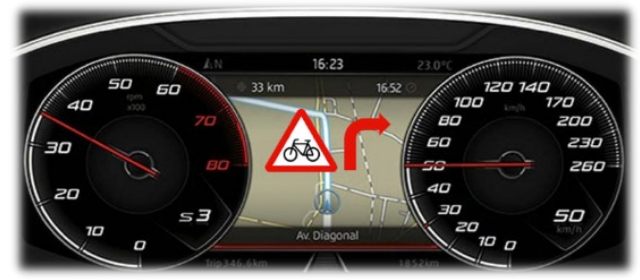

Fig. 11. Cockpit notification in the car.

To show the performance of the different location mechanisms, we performed a test to locate the bicycle using only UWB ranging system and then compare it with the fusion of the three systems GNSS, INS and UWB.

Fig. 12 depicts the result from calculating the bicycle trajectory in the proximity of the intersection using only the distance information provided by the UWB anchors. As it can be observed, in the upper part of the graph, almost all points converge to the expected path, while in the bottom part, a higher dispersion is observed. This is explained by the fact that UWB anchors are placed in the blue area; thus, in the bottom part of the intersection the bicycle has less information to calculate a precise position of the bicycle. Although, the shape of the route followed by the rider can be distinguished along the most part of the path, using only the UWB system for location purposes, it doesn't provide the required performance.

Referring again to Fig. 8 which shows a screenshot of the real-time route followed by the bicycle obtained from the hybrid positioning algorithm combining different information sources in one of the trials. It can be observed how the combined algorithm allows to adequately reproduce with enough precision the full trajectory. It must be noted that in this case, the GPS conditions of the testbed scenario were good since the area was only partially urbanized. Even in this case, the distance information provided by the UWB technology served to perform a finer tuning of the bicycle position providing higher precision, enough to enable the 
proposed C-ITS use case. The arrow indicates the start and the end of the trajectory which included two laps along the roundabout (the bicycle is following the bike line, which does not strictly follow a circle).

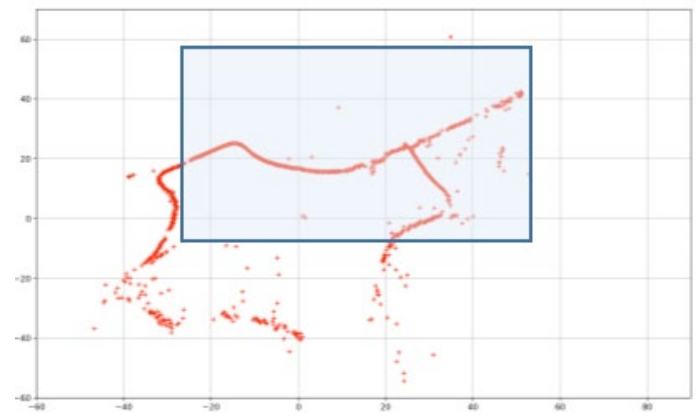

Fig. 12. Route followed by the bicycle using only UWB ranging triangulation.

\section{CONCLUSIONS}

In this paper authors present and implement a solution to improve the safety of vulnerable road users based on the joint usage of vehicle communications and IoT technologies.

The experimental results from the bicycle detection testbed demonstrate how the combination of UWB technologies, inertial sensors and GPS systems can be successfully used to accurately track bicycles in real-time with precisions that can clearly outperform the ones obtained by conventional GPS solutions, especially in urban scenarios where the signal of the GPS might be lost or insufficient. Accurate tracking, together with the ability to acquire and send this information in real-time (every $100 \mathrm{~ms}$ ), makes the solution feasible for deploying C-ITS to warn vehicles from the presence of bicycle riders in their way. Furthermore, the approach could be extended to other vulnerable road users, such as skaters or scooters.

The testbed presented in the paper can be considered a first step in the research of applying UWB technologies to enhance the positioning capabilities of intelligent transport systems. Since UWB allows to measure distances with an accuracy of several centimetres, it might be used, for example, to determine the lane of the road where a vehicle is located and to speed up lane changes when priority vehicles like ambulances are approaching. Integrating UWB technologies with vehicle networks requires to investigate in further aspects such as improving the range and the scalability of the UWB ranging mechanisms and enhancing the adaptability of the positioning algorithms to cover new use cases and different kind of vehicles. Some of these issues will be considered and evaluated in the framework of the 5GCroco H2020 project, which aims to trial Connected, Cooperative and Automated Mobility (CCAM) services in the cross-border corridor along France, Germany and Luxembourg.

\section{ACKNOWLEDGMENTS}

This pilot has been deployed in a partnership with: CTTC, Ficosa, i2CAT, SEAT, Telefónica España and UPC.

Authors also acknowledge Berta Serracanta, Mar Vidal and Julio C. Barrera for their involvement in the implementation of the Bicycle Detection testbed.

Part of the development has been supported by the ERDF the Spanish Government through projects TEC2016-79988-P, TEC2016-76795-C6, AEI/FEDER, UE; by Secretaria d'Universitats i Recerca del Departament d'Empresa i Coneixement de la Generalitat de Catalunya through projects 2017 SGR 00376 and 2017 SGR 1479 and by the EU H2020 5 GCROCO project funded under grant agreement No. 825050 .

\section{REFERENCES}

[1] ETSI TR 102638 V1.1.1. "Intelligent Transport Systems (ITS); Vehicular communications; Basic set of applications; Definitions". ETSI. June 2009.

[2] R. Riebl. "Vanetza framework, Open-source implementation of the ETSI C-ITS protocol stack". Technische Hochschule Ingolstadt. https://github.com/riebl/vanetza [Accessed March 2019].

[3] ETSI EN 302 637-2 v1.4.1 "Intelligent Transport Systems (ITS); Vehicular Communications; Basic Set of Applications; Part 2: Specification of Cooperative. Awareness Basic Service", January 2019.

[4] ETSI EN 302 637-3 v1.3.0 "Intelligent Transport Systems (ITS); Vehicular Communications; Basic Set of Applications; Part 3: Specifications of Decentralized Environmental Notification Basic Service", August 2018.

[5] A. Bazzi, B. M. Masini, A. Zanella and I. Thibault, "On the Performance of IEEE $802.11 \mathrm{p}$ and LTE-V2V for the Cooperative Awareness of Connected Vehicles", IEEE Transactions on Vehicular Technology, vol. 66, no. 11, pp. 10419-10432, November 2017.

[6] R. Molina-Masegosa and J. Gozalvez, "LTE-V for sidelink 5G V2X vehicular communications", IEEE Vehicular Technology Magazine, vol. 12, no. 4, pp. 30-39, December 2017.

[7] "Enhanced real time services for an optimised multimodal mobility relying on cooperative networks and open data (TI-MON) project", European Union's Horizon 2020 research and innovation programme under grant agreement No 636220, 2016, https://timon-project.eu [Accesed March 2019].

[8] European Global Navigation Satellite Systems Agency, "World's first dual-frequency GNSS smartphone hits the market", June 2018, https://www.gsa.europa.eu/newsroom/news/world-s-first-dualfrequency-gnss-smartphone-hits-market [Accesed March 2019].

[9] M. S. Grewal, L. R. Weill, and A. P. Andrews, "Global Positioning Systems, Inertial Navigation and Integration," 2nd Ed., John Wiley \& Sons, Inc., Hoboken, New Jersey, 2007.

[10] P. D. Groves,Principles of GNSS, "Inertial and Multisensor Integrated Navigation Systems", 2nd Ed., Artech House,Norwood, United States, 2013.

[11] J. Arribas, A. Moragrega, C. Fern' andez-Prades, and P. Closas, "Lowcost GNSS/INS/Odometric sensor fusion platform for ground intelligent transportation systems", ION GNSS 2017, Portland (USA), September 2017.

[12] J. Arribas, M. Navarro, A. Moragrega, D. Calero, E. Fernández, J. VilàValls, C. Fernández-Prades, "A Technology-agnostic GNSS/INS Realtime Sensor Fusion Platform with Ultra Wide Band Cooperative Distance Measurements for Terrestrial Vehicle Navigation", 31th International Technical Meeting of The Satellite Division of the Institute of Navigation (ION GNSS+ 2018), Miami (USA), 24-28 September 2018 . 